\title{
The core clock gene Per1 phases molecular and electrical circadian rhythms in SCN neurons
}

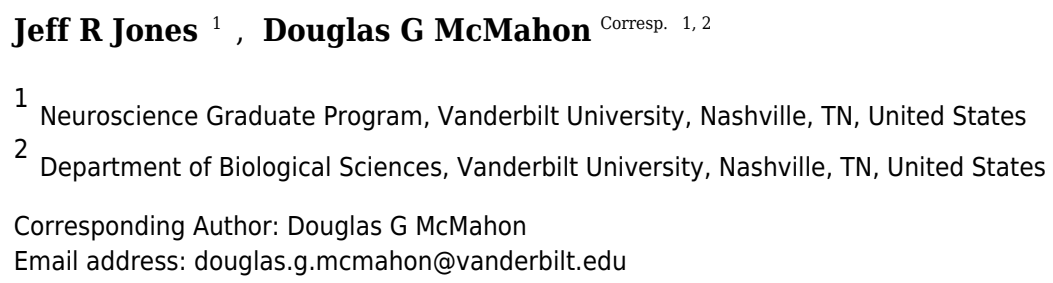

The brain's biological clock, the suprachiasmatic nucleus (SCN), exhibits endogenous 24hour rhythms in gene expression and spontaneous firing rate; however, the functional relationship between these neuronal rhythms is not fully understood. Here, we used a Per1::GFP transgenic mouse line that allows for the simultaneous quantification of molecular clock state and firing rate in SCN neurons to examine the relationship between these key components of the circadian clock. We find that there is a stable, phased relationship between E-box-driven clock gene expression and spontaneous firing rate in SCN neurons and that these relationships are independent of light input onto the system or of $\mathrm{GABA}_{A}$ receptor-mediated synaptic activity. Importantly, the concordant phasing of gene and neural rhythms is disrupted in the absence of the homologous clock gene Per1, but persists in the absence of the core clock gene Per2. These results suggest that Per1 plays a unique, non-redundant role in phasing gene expression and firing rate rhythms in $\mathrm{SCN}$ neurons to increase the robustness of cellular timekeeping. 
1 The core clock gene Per1 phases molecular and electrical circadian 2 rhythms in SCN neurons.

3

4 Jeff R. Jones ${ }^{1 *}$, Douglas G. McMahon ${ }^{1,2}$

5

7

8

9

10

11

12

13

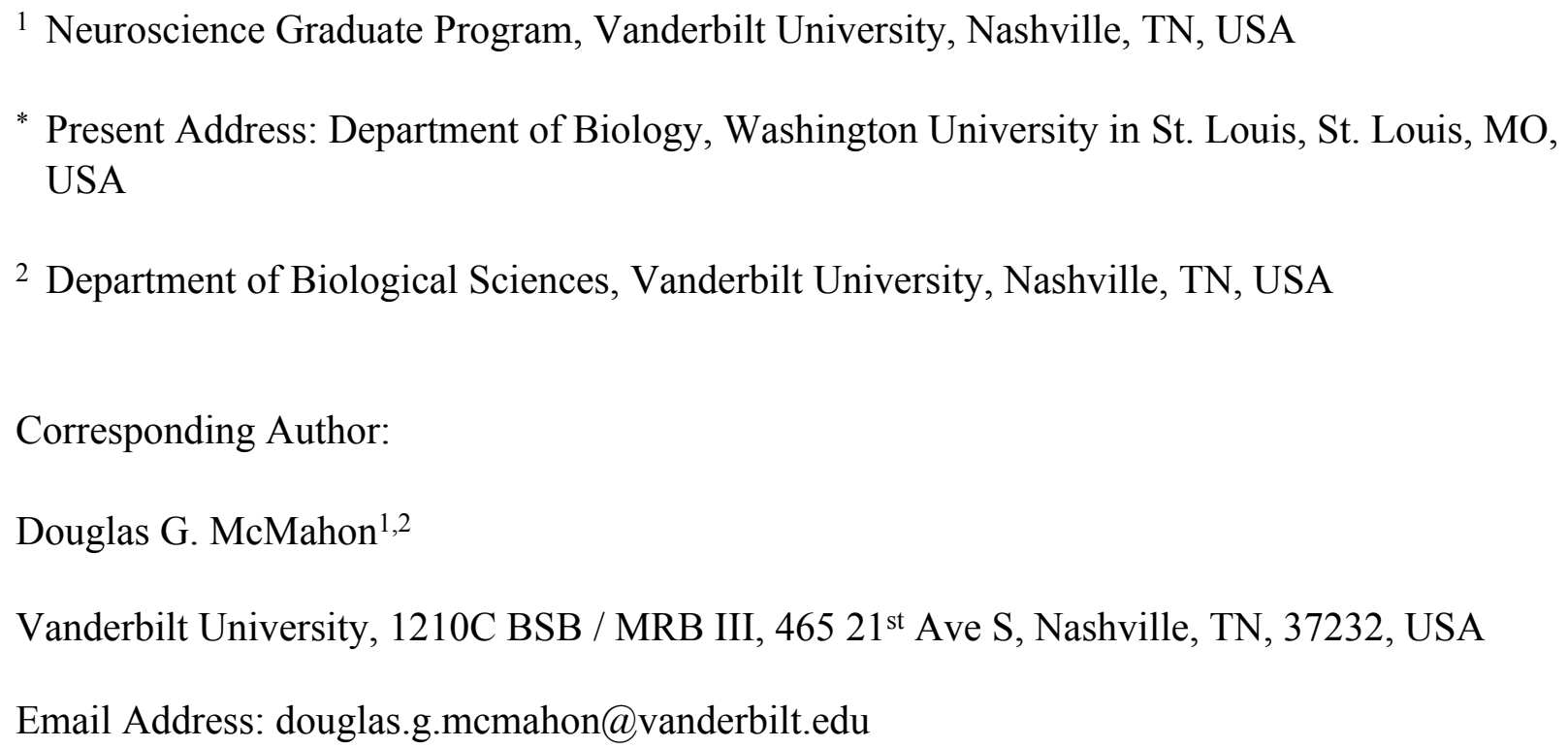


22 ABSTRACT

23 The brain's biological clock, the suprachiasmatic nucleus (SCN), exhibits endogenous 24-hour

24 rhythms in gene expression and spontaneous firing rate; however, the functional relationship

25 between these neuronal rhythms is not fully understood. Here, we used a Per $1:$ GFP transgenic

26 mouse line that allows for the simultaneous quantification of molecular clock state and firing rate

27 in SCN neurons to examine the relationship between these key components of the circadian

28 clock. We find that there is a stable, phased relationship between E-box-driven clock gene

29 expression and spontaneous firing rate in SCN neurons and that these relationships are

30 independent of light input onto the system or of $\mathrm{GABA}_{\mathrm{A}}$ receptor-mediated synaptic activity.

31 Importantly, the concordant phasing of gene and neural rhythms is disrupted in the absence of

32 the homologous clock gene Perl, but persists in the absence of the core clock gene Per2. These results suggest that Perl plays a unique, non-redundant role in phasing gene expression and

34 firing rate rhythms in SCN neurons to increase the robustness of cellular timekeeping. 


\section{INTRODUCTION}

Understanding how gene signaling networks influence the activity of neurons and circuits that control behavior is an essential problem in neuroscience. Daily changes in physiology and behavior in mammals are driven by the suprachiasmatic nucleus ( $\mathrm{SCN}$ ), a network of neurons exhibiting endogenous rhythms in gene expression and firing rate in isolation (Colwell, 2011). A key unsolved question in circadian neurobiology is how these neuronal rhythms interact to form a coherent pacemaker. Circadian gene expression rhythms in SCN neurons are driven by an autoregulatory transcriptional / translational feedback loop (TTFL) comprised of the core clock genes Bmall, Clock, Per1/2, and Cryl/2. Furthermore, circadian firing rate rhythms are produced by a collection of intrinsic ionic currents that allow SCN neurons to spontaneously fire action potentials and, importantly, modulate their firing rates so that they fire at up to 6 to $12 \mathrm{~Hz}$ during the day and 0 to $2 \mathrm{~Hz}$ at night. The central role of the TTFL in driving circadian electrical activity has been supported by studies in which the cycling of the molecular clock was halted by the elimination of critical clock genes (Liu et al., 1997; Herzog et al., 1998; Nakamura et al. 2002; Albus et al., 2002). However, blocking action potentials in SCN neurons and in clock neurons during development in Drosophila disrupts gene cycling (Yamaguchi et al., 2003; Nitabach et al., 2002), and optogenetically driving SCN neuron firing rates can reset the molecular clockworks (Jones et al., 2015). Thus, the relationship between clock neuron electrical activity and the molecular clockworks is likely bidirectional.

A fundamental gap in our knowledge is therefore understanding how the circadian molecular clockworks is linked to firing rate rhythms in the SCN. To investigate this, we have used our Per 1::GFP transgenic mouse line in which sequences from the Per 1 gene promoter that contain 
58

both E-box enhancer elements for CLOCK/BMAL1-driven transcription as well as CRE elements for CREB-driven induction drive a short half-life version of enhanced green fluorescent protein (d2EGFP; Kuhlman et al., 2000). Importantly, there is both a high concordance of d2EGFP expression with Per1 mRNA and PER1 protein expression in the SCN of these mice and congruous regional distributions and rhythms which suggests that the d2EGFP construct in Per 1::GFP mice reports native Perl gene expression with high fidelity (LeSauter et al. 2003). Using this artificial clock-controlled gene we have previously shown that the degree of activation of this construct correlates with firing rate in individual SCN neurons during the day phase of circadian cycling and following a phase-resetting light pulse at night (Kuhlman et al., 2003;

Quintero et al., 2003). This correlation and additional experiments with circadian reporters suggest that a fixed phase relationship may exist between the molecular clockworks and circadian electrical activity (Colwell, 2011). Intriguingly, neuropeptide resetting of SCN neuron firing rate requires the translation of the native Perl gene, which suggests a functional role for Perl in this relationship (Gamble et al., 2007; Kudo et al., 2013).

In order to study Perl's role as a potential link between molecular and electrical circadian rhythms, we crossed our Per1::GFP mice with $\mathrm{Per}^{1^{-/}}$, Per2 ${ }^{-/}$, and $\mathrm{Per}^{--}$; $\mathrm{Per}^{2-/}$ mice (Bae et al. 2001) that had been bred congenic on a C57BL/6J background (Pendergast et al. 2009; 2010) to yield Per knockout mice that express d2EGFP as a transcriptional reporter of the molecular clockworks. Importantly, the GFP construct is still rhythmically expressed in $\operatorname{Per}^{-/}$Per $1:$ GFP animals because its production is regulated by the E-box and CRE elements in the Perl promoter sequences in the transgene (Kuhlman et al. 2000). In vivo, the knockout of Per1 or Per2 individually in mice on a congenic $\mathrm{C} 57 \mathrm{BL} / 6 \mathrm{~J}$ background results in rhythmic wheel-running 
81 behavior that is similar to that of wild-type mice (Pendergast et al. 2009; Pendergast et al. 2010a;

$82 \mathrm{Xu}$ et al. 2007), while $\mathrm{Per}{ }^{-/-} ; \mathrm{Per}^{-/-}$double knockout mice on a C57BL/6J background are

83 behaviorally arrhythmic in constant darkness (Pendergast and Yamazaki 2011). In vitro,

84 however, SCN cultures from $\mathrm{Perl}^{-/}$mice exhibit weakened or variable rhythms in circadian gene

85 expression as read out by PER2::LUCIFERASE that can, in some cases, be enhanced by a media change (Liu et al. 2007; Pendergast et al. 2010; Ruan et al. 2012). The period of Perl-luc gene expression rhythms in $\mathrm{Per}^{2 /-} \mathrm{SCN}$ cultures are markedly shorter than wild-type rhythms (Pendergast et al. 2010a). Importantly, in vivo multi-unit neural activity from $\mathrm{Perl}^{-/-}$mice is rhythmic, which may explain the discrepency between the weakly-rhythmic molecular clockworks observed in vitro and robustly rhythmic behavioral rhythms observed in vivo in these mice (Takasu et al. 2013).

Here we report a consistent, almost synchronous, phase relationship between the molecular clockworks and circadian electrical activity throughout the 24 hour circadian day assayed in individual SCN neurons of in vitro SCN slices. Knockout of Perl alters this relationship, delaying molecular activity relative to firing rate rhythms by about 6 hours such that there is an approximately 90 degree phase angle between them. Importantly, this effect is specific to Perl, as the close synchrony between circadian gene expression and firing rate rhythms is preserved when the clock gene Per2 is knocked out. Taken together these results identify Perl as a clock gene that phases the molecular clockworks and circadian electrical activity into reinforcing synchrony. 


\section{Animals and housing}

Experiments were performed using male and female Per $1:$ d2EGFP ("Perl::GFP"; Kuhlman et

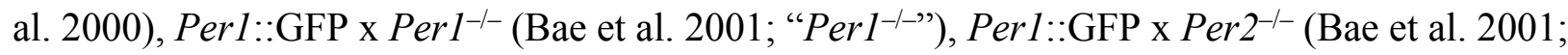

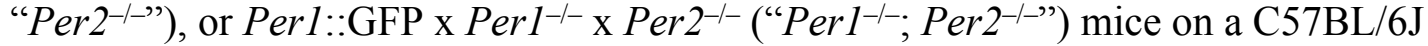
background, 1-3 months of age. Animals were provided with food and water ad libitum and were housed in single-sexed cages of no more than five animals from weaning until experimental use on a 12:12 light/dark (LD) cycle, or, for some experiments, were housed in constant darkness (DD) for at least two weeks before use. All animal care and experimental procedures were conducted in concordance with Vanderbilt University's Institutional Animal Care and Use Committee guidelines.

\section{Behavioral analysis}

Wheel-running activity from mice housed in DD was monitored and recorded in 5 minute bins using ClockLab software (Actimetrics, Evanston, IL). Time of activity onset (defined as circadian time 12 [CT 12]) was determined using ClockLab Analysis Software. As Per1 ${ }^{-/-}$; Per2 ${ }^{\text {I- }}$ mice are behaviorally arrhythmic, CT could not be defined for this group of mice.

\section{Slice preparation}

Mice housed in LD were killed by cervical dislocation without anesthesia in ambient light for dissections occurring between ZT (Zeitgeber time) 0 to 12, where ZT 0 is defined as the time of lights on and ZT 12 is defined as the time of lights off. For dissections occurring between ZT 12-24 for mice housed in LD, or for all dissections from mice housed in DD, mice were killed by cervical dislocation without anesthesia under dim red light ( $<1$ lux). Importantly, dissections 
127 occurring in the dark phase have been shown not to affect the phase of electrical activity rhythms

128 in the SCN (vanderLeest et al., 2009). After dissection, brains were quickly removed and

129 blocked in cold, oxygenated $95 \% \mathrm{O}_{2}-5 \% \mathrm{CO}_{2}$ dissecting solution (in $\mathrm{mM}$ : $114.5 \mathrm{NaCl}, 3.5 \mathrm{KCl}$,

$1301 \mathrm{NaH}_{2} \mathrm{PO}_{4}, 1.3 \mathrm{MgSO}_{4}, 2.5 \mathrm{CaCl}_{2}, 10 \mathrm{D}$-glucose, and $\left.35.7 \mathrm{NaCHO}_{3}\right)$. SCN slices $(200 \mu \mathrm{m})$

131 were cut coronally on a vibroslicer (World Precision Instruments, Sarasota, FL) at $4-10^{\circ} \mathrm{C}$ and

132 transferred directly to an open recording chamber continually superfused with warmed (35 \pm

$1330.5^{\circ} \mathrm{C}$ ) extracellular solution (in mM: $124 \mathrm{NaCl}, 3.5 \mathrm{KCl}, 1 \mathrm{NaH}_{2} \mathrm{PO}_{4}, 1.3 \mathrm{MgSO}_{4}, 2.5 \mathrm{CaCl}_{2}, 10$

134 D-glucose, and $26 \mathrm{NaCHO}_{3}$ ). Slices were allowed to recover for 1 hour before recording.

135

136

137

138

139

140

141

142

143

144

145

146

147

148

149

\section{Electrophysiological recording and imaging}

SCN neurons were visualized using a Leica DMLFS microscope (Leica Microsystems, Buffalo Grove, IL) equipped with near-infrared (IR)-differential interference contrast and fluorescence optics. For loose-patch recordings, patch electrodes (4-6 M $\Omega$ ) pulled from glass capillaries (WPI) on a multistage puller (DMZ; Zeitz, Martinsried, Germany) were filled with extracellular solution. Spontaneous action potential recordings ( $\sim 5$ minutes in duration) from neurons sampled throughout the SCN were obtained with an Axopatch 200B amplifier (Molecular Devices, Sunnyvale, CA) and monitored online with pClamp 10.0 software (Molecular Devices). Recordings were obtained in gap-free mode throughout the day were sampled at $10 \mathrm{kHz}$, and were filtered online at $1 \mathrm{kHz}$. Loose-patch seal resistances ranged from 10-30 M $\Omega$. Slices were used for no more than 6 hours after dissection. Immediately after cessation of electrophysiological recording, an image of the recorded neuron was captured with an exposure time of one second using HCImage acquisition software (Hamamatsu; Bridgewater, NJ) with a cooled CCD camera (Hamamatsu) and an EGFP filter set. All recordings were confirmed to be 
150 from $\mathrm{GFP}^{+}$neurons by aligning digital images of the same neuron taken under near-IR and GFP 151 fluorescence illumination.

152

153

\section{Image and electrophysiology analysis}

Image analysis was performed based on methods described in Kuhlman et al. (2003), using ImageJ software with 16-bit digitization. Fluorescence was reported as the intensity of a region of interest containing the cell body divided by the background fluorescence to normalize for differences in baseline fluorescence across preparations and fields. Background fluorescence was defined as the average pixel intensity of two local measurements next to the recorded neuron and the total frame (1024 x 1024 pixels). Average firing rate for the recording period was calculated using Clampex software (Molecular Devices).

\section{RESULTS}

To assay the phase relationship between E-box-driven clock gene expression and spontaneous firing rate in the SCN, we performed visually-targeted loose-patch recording of individual $\mathrm{GFP}^{+}$ SCN neurons in coronal SCN slices from Per1::GFP mice (Kuhlman et al., 2000) sampling around the clock. We found that both E-box-driven clock gene expression as measured by GFP fluorescence intensity and spontaneous firing rate showed temporal variations consistent with ongoing rhythms at a population level (Fig. 1a). The population of recorded SCN neurons exhibited a peak in spontaneous firing rate as determined by a Cosinor fit at around Zeitgeber Time (ZT) 7 and a peak in GFP fluorescence intensity at ZT 9, where ZT 0 is defined as the time of lights on. Thus, observed rhythms in firing rate and gene expression were closely aligned, 
173 with firing rate phase leading by about two hours. When the time to translate and fold d2EGFP

174 of approximately two hours is accounted for ( $\mathrm{Li}$ et al. 1998), the firing rate rhythm would be

175 projected to be essentially synchronous with the transcriptional rhythm. To further analyze the

176 relationship between GFP fluorescence intensity and spontaneous firing rate we plotted these

177 parameters for each recorded neuron (Fig. 1b). Overall, fluorescence intensity ranged from 1.0

178 to $1.25 \mathrm{x}$ background, while firing rates varied from 0 to $12 \mathrm{~Hz}$. Individual SCN neurons showed

179 a range of variability in both firing rates and fluorescence intensities, and in aggregate there was

180 a significant positive linear correlation between Perl promoter activiation as read out by GFP

181 fluorescence intensity and spontaneous firing rate assayed within individual SCN neurons over

182 the course of the 24 hour sampling (Fig. 1b; $\mathrm{n}=249$ cells, 34 mice; Pearson's $r, r^{2}=0.4504, p<$

183 0.001). Although this relationship can be approximated as linear, it is likely to more complex and

184 cyclical in nature (see Fig. 5 and Discussion).

185

186

187

188

189

190

191

192

193

194

To test whether these results were due to circadian rhythms or diurnal light-driven responses we performed experiments on SCN from mice housed in constant darkness. Both GFP fluorescence intensity and spontaneous firing rate remained rhythmic (Fig. 1c), and the positive correlation between GFP fluorescence intensity and spontaneous firing rate in individual neurons persisted in constant darkness (Fig. 1d). Finally, most SCN neurons are GABAergic (Wagner et al., 1997) and therefore GABA transmission represents much of the ongoing rapid synaptic transmission in the nucleus. We found that when the $\mathrm{GABA}_{\mathrm{A}}$-receptor blocker GABAzine was applied to SCN neurons from mice housed under a normal light cycle, GFP fluorescence intensity and spontaneous firing rate remained rhythmic at the population level (Fig. 1e). Likewise, the 
195 positive correlation between GFP fluorescence intensity and spontaneous firing rate in individual

196 neurons again persisted in the presence of this blocker (Fig. 1f).

197

198 To determine if this observed population- and single-cell stable phase relationship between E-

199

200

201

202

203

204

205

206

207

208

209

210

211

212

213

214

215

216

217

box-driven gene expression and spontaneous firing rate persisted in the absence of the core clock gene Perl, we recorded individual $\mathrm{GFP}^{+} \mathrm{SCN}$ neurons from $P$ er $1^{-/}$; Perl::GFP mice sampled around the clock. Importantly, in these mice, the E-box-driven production of GFP still acts as a reporter of the molecular clockworks since the reporter gene is driven by a BMAL1/CLOCK heterodimer even in the absence of the native Perl gene. We found that at the population level both E-box-driven clock gene expression as measured by GFP fluorescence intensity and spontaneous firing rate exhibited statistically significant time series variations, however, their phase relationship was radically changed (Fig. 2a). SCN neurons from $\mathrm{Per}^{-/-}$mice exhibited a peak in GFP fluorescence intensity as determined by a Cosinor fit that was significantly delayed (ZT 12) compared to the peak of spontaneous firing rate (ZT 5) - a difference of about 7 hours.

Strikingly, the robust correlation between GFP fluorescence intensity and spontaneous firing rate we observed in individual SCN neurons from wild-type mice was completely lost in individual Per $1^{-/-}$SCN neurons (Fig. 2b). This is an outcome of the change in relative phases of the firing rate and molecular rhythms to an approximately 6 hour (i.e., 90 degree) difference. In SCN neurons from $\mathrm{PerI}^{-/-}$mice housed in constant darkness, the correlation between GFP fluorescence intensity and spontaneous firing rate in individual cells remained absent (Fig. 2d); at the population level, GFP fluorescence intensity remained rhythmic, and the temporal profile of the spontaneous firing rate was was below the significance level for rhythmicity $(p=0.067$; Fig. 2c). 
219 To determine if the altered population-level and abolished single-cell phase relationship between

220

221

222

223

224

225

226

227

228

229

230

231

232

233

234

235

236

237

238

239

240

E-box-driven gene expression and spontaneous firing rate we observed in $\mathrm{Perl}^{-/} \mathrm{SCN}$ neurons

was specific to the loss of Perl, we recorded from individual $\mathrm{GFP}^{+} \mathrm{SCN}$ neurons in $\mathrm{SCN}$ slices

from $\operatorname{Per}^{-/-}$; Per $1::$ GFP mice sampled throughout the day. Both E-box-driven clock gene

expression as measured by GFP fluorescence intensity and spontaneous firing rate were rhythmic

at a population level (Fig. 3a). Similar to wild type, and in contrast to Perl $^{-/}$SCN neurons, the

population of recorded SCN neurons in $\mathrm{Per}^{-/-}$mice exhibited a peak in spontaneous firing rate at about ZT 7 and a peak in GFP fluorescence intensity at about ZT 9. Critically, the single-cell

correlation between GFP fluorescence intensity and spontaneous firing rate was also preserved in the absence of Per2 (Fig. 3b). In SCN neurons from Per $2^{-1-}$ mice housed in constant darkness, we continued to observe stable population-level and single-cell phase relationships between GFP fluorescence intensity and spontaneous firing rate (Fig. 3c,d). This single-cell correlation was abolished when Perl was concurrently knocked out in SCN neurons from $P e r 1^{-1-}$ Per2 ${ }^{-{ }^{--}}$mice (Fig. 4).

\section{DISCUSSION}

To investigate the relationship between gene expression rhythms and circadian electrical activity in the $\mathrm{SCN}$, we performed firing rate recording and real-time fluorescence imaging of the state of the molecular clockworks in Perl::GFP ${ }^{+} \mathrm{SCN}$ neurons throughout the circadian day. We found that E-box-driven gene expression and spontaneous firing rate rhythms were closely aligned in individual Per $1::$ GFP SCN neurons, with firing rate rhythms consistently phase leading gene expression rhythms by about two hours. Given the estimated two hour time course for the 
translation and folding of d2EGFP ( $\mathrm{Li}$ et al. 1998), this suggests that spontaneous firing and the activation of the Perl promoter are essentially synchronous. In SCN neurons from $\mathrm{Perl}^{-/-}$mice this relationship is altered such that gene expression rhythms are significantly delayed in relationship to firing rate rhythms. Importantly, in the absence of Perl there is no longer a predictive correlation between gene expression and neural activity in individual SCN neurons. However, in SCN neurons from $P e r 2^{-/-}$mice the phase relationship between circadian rhythms in gene expression and firing rate is intact. These results therefore demonstrate that Perl is necessary for maintaining a synchronous phase relationship between the molecular clockworks and membrane electrical activity.

The finding that there exists a stable, reinforcing phase relationship between E-box driven gene expression and spontaneous firing rate across an entire circadian day expands upon previous studies showing that there is a correlation between these two components of the circadian clock at midday and after a phase-resetting light pulse at night (Kuhlman et al., 2003; Quintero et al., 2003). Here we not only show that this correlation persists in neurons sampled across 24 hours, but that gene expression rhythms consistently aligned in phase with spontaneous firing rate rhythms. Importantly, Per $1:$ GFP expression in a wild-type animal peaks at approximately the same time as the peak of native PER1 protein expression (LeSauter et al., 2003; which peaks approximately two to four hours after the peak of native Perl mRNA expression), and our observed time of peak spontaneous firing rate is also consistent with that found in other studies (Meijer et al., 1997; but see Belle et al., 2009). Thus, by simultaneously measuring the state of the molecular clock and electrical activity in individual neurons within SCN slices, we were able to determine the instantaneous circadian relationship between these two key components of the 
264 circadian clock in individual SCN neurons. This newly established within-cell relationship 265 therefore predicts that an increase in firing rate precedes an increase in translation of PER1 266 (although it may occur virtually concurrently with the transcription of Perl itself). The canonical 267 multi-component model of the mammalian circadian clock supposes that firing rate is solely an

output of the state of the molecular clock; however, these results suggest that firing rate, peaking $\sim 2$ hours before the peak of GFP fluorescence (and thus the peak of PER1 translation), is likely an input onto the molecular clock, as also suggested by recent results demonstrating that optogenetic manipulation of clock neurons can reset the molecular clockworks (Jones et al., 2015).

Our $\mathrm{Perl}^{-/}$data suggest that the alignment of SCN neuronal firing rate and the molecular clockworks in a reinforcing phase relationship increases the robustness of both oscillations. Knocking out Perl results in a 90 degree phase relationship between these components of the circadian clock that weakens molecular rhythms as evidenced by a decrease in the amplitude of Per 1::GFP fluorescence rhythms in LD and a lack of Per1::GFP fluorescence rhythms in DD. Interestingly, the weakened neural and molecular rhythms we observe in $\mathrm{Perl}^{-/-} \mathrm{SCN}$ neurons may account for the high-amplitude phase response curve to light observed in vivo in Perl $1^{-/}$ mice, as a weaker central clock is shifted more easily (Pendergast et al. 2010b).

\section{The $\mathrm{Perl}^{-/-}$and $\mathrm{Per}^{-/-}$data presented here are in agreement with previously reported data from} these mice on a congenic C57BL/6J background. $\mathrm{Perl}^{-/-}$mice are behaviorally rhythmic (Pendergast et al. 2009), exhibit in vivo SCN firing rate rhythms (Takasu et al. 2013) and, importantly, their in vitro SCN molecular rhythms as assayed by Perl-luc are less robustly 
287 288 289 290

rhythmic for one or more peaks in vitro with a delayed phase (Pendergast et al. 2009); similarly, Per2-/- mice have intact behavioral and molecular rhythms (Pendergast et al 2010). In the present study, SCN neurons in SCN slices from our $\mathrm{Per}^{-/-}$mice as a population exhibit somewhat depressed firing rate rhythms and rhythmic but dampened and phase-delayed Per 1::GFP fluorescence rhythms, while SCN neurons from our Per2-/- mice exhibit firing rate and fluorescence rhythms that are comparable to those of wild-type controls.

How, though, could clock neuron electrical activity, an ostensible output of the molecular clock, also affect the state of the molecular clock itself? The most likely candidate for this connection is yet another component of neuronal circadian rhythms - daily oscillations in intracellular second messengers, including $\mathrm{Ca}^{2+}$ and cAMP (Brancaccio et al., 2013). Some SCN neurons can exhibit firing rate rhythms on genetic backgrounds in which gene cycling is absent, suggesting that the ionic mechanisms at the membrane can oscillate in a circadian manner without an intact TTFL (Nakamura et al., 2002). Similarly, genetic or physiological blockade of the firing rate rhythm in Drosophila (dORK channel) or mouse (TTX) leads to run-down of clock gene cycling in clock neurons (Nitabach et al., 2002; Yamaguchi et al., 2003). This is likely due to blunting of cellular calcium rhythms as chelation of extracellular $\mathrm{Ca}^{2+}$ blunts gene expression rhythms (Lundkvist et al., 2005). Interestingly, a recent study using antisense oligodeoxynucleotides to knock down Perl in SCN neurons found that this treatment not only transiently alters firing rate but also reduces intracellular levels of calcium and levels of calcium-activated potassium currents (Kudo et al. 2015). Finally, another intracellular messenger, cAMP, which in SCN neurons is primarily controlled by VIPergic intercellular communication through VPAC2 receptors, is also critical for 
sustained gene and firing rate rhythms (Aton et al. 2005; Atkinson et al. 2011; Cutler et al. 2003).

We suggest that Perl, with its rapid high-amplitude CRE-driven responses (Tischkau et al., 2003), mediates resonant phasing of firing rate and E-box-driven transcription in both wild-type and $P e r 2^{-/-}$SCN neurons. This phasing likely occurs through the rapid response of Perl to CREB/CRE signals from upstream membrane-driven $\mathrm{Ca}^{2+}$ and/or cAMP (Brancaccio et al., 2013) and subsequent downstream inhibition of potassium currents through FDR or other potassium channels (Kuhlman and McMahon, 2004; Kudo et al., 2011). Without Per1, the rhythms in firing rate and gene expression are displaced out of near-synchronous phase to a 90 degree relative phase where they are no longer reinforcing. As a consequence, single-cell SCN oscillations in $\mathrm{Perl}^{-/-}$mice are weakened (Pendergast et al., 2009). In situ, in the fully intact SCN network, this effect can be rescued through SCN network coupling; however, in dispersed SCN neurons, in fibroblasts, and in peripheral tissue cells that lack the SCN's inherent network coupling, knocking out Perl strongly disrupts the function of the molecular clock (Liu et al., 2007). A limitation of our current study is that it was performed in SCN slices, which represent a semi-intact network. Thus, we cannot on the basis of this data determine which aspects of the altered SCN neuron function in $\mathrm{Per}^{-/-}$mice are due to cell-autonomous effects and which may be due to network compensation. Further experiments with dispersed SCN neurons are necessary to resolve this issue.

\section{Intriguingly, when the relationship between firing rate and the molecular clock in individual} SCN neurons from Per $1:$ GFP, Perl $^{-/}$, and Per2-/- mice is analyzed over time, a cyclical pattern 
332 emerges (Fig. 5). Although we there was a significant linear correlation between the overall

333 firing rate and overall E-box driven gene expression in Per $1::$ GFP or Per2 ${ }^{-/-}$SCN neurons and a

334 loss of that correlation in $\mathrm{Perl}^{-/-} \mathrm{SCN}$ neurons, plotting these variables as 1 hour averages over

33524 hours reveals a non-linear, cyclical trajectory. This indicates that the relationship between

336 firing rate and the molecular clock over time is cyclical rather than linear.

337

338 Clock genes, including Perl, are widely expressed in the nervous system and therefore may be

339 key regulators of neuronal activity in many brain circuits. Indeed, $\mathrm{Per}^{-/-}$mice show alterations

340 in many forms of neural plasticity including cocaine sensitization, LTP, and memory processing

341 (Abarca et al., 2002; Rawashdeh et al., 2014). These results therefore expand upon the role of

342 Perl in the generation of circadian rhythms and demonstrate that Perl in and of itself is key for

343 the phasing of gene expression and neural activity in individual neurons. 


\section{REFERENCES}

Abarca C, Albrecht U, Spanagel R (2002). Cocaine sensitization and reward are under the influence of circadian genes and rhythm. Proc Natl Acad Sci USA 99:9026-9030.

Albus H, Bonnefont X, Chaves I, Yasui A, Doczy J, van der Horst GT, Meijer JH (2002) Cryptochrome-deficient mice lack circadian electrical activity in the suprachiasmatic nuclei. Curr Biol 12:1130-1133.

Aton SJ, Colwell CS, Harmar AJ, Waschek J, Herzog ED (2005) Vasoactive intestinal polypeptide mediates circadian rhythmicity and synchrony in mammalian clock neurons. Nat Neurosci 8:476-483.

Atkinson SE, Maywood ES, Chesham JE, Wozny C, Colwell CS, Hastings MH, Williams SR (2011) Cyclic AMP signaling control of action potential firing rate and molecular circadian pacemaking in the suprachiasmatic nucleus. J Biol Rhythms 26:210-220.

Bae K, Jin X, Maywood ES, Hastings MH, Reppert SM, Weaver DR (2001) Differential functions of mPer1, mPer2, and mPer3 in the SCN circadian clock. Neuron 30:525-36.

Belle MD, Diekman CO, Forger DB, Piggins HD (2009) Daily electrical silencing in the mammalian circadian clock. Science 326:281-284.

Brancaccio M, Maywood ES, Chesham JE, Loudon AS, Hastings MH (2013) A Gq-Ca2+ axis controls circuit-level encoding of circadian time in the suprachiasmatic nucleus. Neuron 78:714728.

Colwell CS (2011) Linking neural activity and molecular oscillations in the SCN. Nat Rev Neurosci 12:553-569.

Cutler DJ, Haraura M, Reed HE, Shen S, Sheward WJ, MOrrison CF, Marston HM, Harmar AJ, Piggins HD (2003) The mouse VPAC2 receptor confers suprachiasmatic nuclei cellular rhythmicity and responsiveness to vasoactive intestinal polypeptide in vitro. Eur J Neurosci 17:197-204.

Gamble KL, Allen GC, Zhou T, McMahon DG (2007) Gastrin-releasing peptide mediates lightlike resetting of the suprachiasmatic nucleus circadian pacemaker through cAMP response element-binding protein and Per1 activation. J Neurosci 27:12078-12087.

Herzog ED, Takahashi JS, Block GD (1998) Clock controls circadian period in isolated suprachiasmatic nucleus neurons. Nat Neurosci 1:708-713.

Jones JR, Tackenberg MC, McMahon DG (2015) Manipulating circadian clock neuron firing rate resets molecular circadian rhythms and behavior. Nat Neurosci 18:373-375. 
Kudo T, Tahara Y, Gamble KL, McMahon DG, Block GD, Colwell CS (2013) Vasoactive intestinal peptide produces long-lasting changes in neural activity in the suprachiasmatic nucleus. J Neurophysiol 110:1097-1106.

Kudo T, Loh DH, Dika K, Cara C, Colwell CS (2011) Fast delayed rectifier potassium current: critical for input and output of the circadian system. J Neurosci 31:2746-2755.

Kudo T, Block GD, Colwell CS (2015) The circadian clock gene Per 1 connects the molecular clock to neural activity in the suprachiasmatic nucleus. ASN Neuro 7:1-14.

Kuhlman SJ, Silver R, LeSauter LJ, Bult-Ito A, McMahon DG (2003) Phase resetting light pulses induce Per 1 and persistent spike activity in a subpopulation of biological clock neurons. $\mathrm{J}$ Neurosci 23:1441-1450.

Kuhlman SJ, Quintero JE, McMahon DG (2000) GFP fluorescence reports Period 1 circadian gene regulation in the mammalian biological clock. Neuroreport 11:1479-1482.

Kuhlman SJ, McMahon DG (2004) Rhythmic regulation of membrane potential and potassium current persists in SCN neurons in the absence of environmental input. Eur J Neurosci 20:11131117.

LeSauter J, Yan L, Vishnubhotla B, Quintero JE, Kuhlman SJ, McMahon DG, Silver R (2003). A short half-life GFP mouse model for analysis of suprachiasmatic nucleus organization. Brain Res 964:279-287.

Li X, Zhao X, Fang Y, Jiang X, Duong T, Fan C, Huang CC, Kain SR (1998) Generation of destabilized green fluorescent protein as a transcription reporter. J Biol Chem 273:34970-5.

Liu AC, Welsh DK, Ko CH, Tran HG, Zhang EE, Priest AA, Buhr ED, Singer O, Meeker K, Verma IM, Doyle FJ, Takahashi JS, Kay SA (2007) Intercellular coupling confers robustness against mutations in the SCN circadian clock network. Cell 129:605-616.

Liu C, Weaver DR, Strogatz SH, Reppert SM (1997) Cellular construction of a circadian clock: period determination in the suprachiasmatic nuclei. Cell 91:855-860.

Lundkvist GB, Kwak Y, Davis EK, Tei H, Block GD (2005) A calcium flux is required for circadian rhythm generation in mammalian pacemaker neurons. J Neurosci 25:7682-7686.

Meijer JH, Schaap J, Watanabe K, Albus H (1997) Multiunit activity recordings in the suprachiasmatic nuclei: in vivo versus in vitro models. Brain research 753:322-327.

Nakamura W, Honma S, Shirakawa T, Honma K (2002) Clock mutation lengthens the circadian period without damping rhythms in individual SCN neurons. Nat Neurosci 5:399-400.

Nitabach MN, Blau J, Holmes TC (2002) Electrical silencing of Drosophila pacemaker neurons stops the free-running circadian clock. Cell 109:485-495. 
434
Pendergast JS, Friday RC, Yamazaki S (2009) Endogenous rhythms in Period1 mutant suprachiasmatic nuclei in vitro do not represent circadian behavior. J Neurosci 29:14681-14686.

Pendergast JS, Friday RC, Yamazaki S (2010) Distinct functions of Period2 and Period3 in the mouse circadian system as revealed by in vitro analysis. PLoS One 5:e8552.

Pendergast JS, Friday RC, Yamazaki S (2010) Photic entrainment of Period mutant mice is predicted from their phase response curves. J Neurosci 30:12179-12184.

Pendergast JS, Yamazaki S (2011) Masking responses to light in period mutant mice. Chronobiol Int 28:657-63.

Quintero JE, Kuhlman SJ, McMahon DG (2003) The biological clock nucleus: a multiphasic oscillator network regulated by light. J Neurosci 23:8070-8076.

Rawashdeh O, Jilg A, Jedlicka P, Slawska J, Thomas L, Saade A, Schwarzacher SW, Stehle JH (2014) PERIOD1 coordinates hippocampal rhythms and memory processing with daytime. Hippocampus 6:712-723.

Ruan GX, Gamble KL, Risner ML, Young LA, McMahon DG (2012) Divergent roles of clock genes in retinal and suprachiasmatic nucleus circadian oscillators. PLoS One 7:e38985.

Takasu NN, Pendergast JS, Olivas CS, Yamazaki S, Nakamura W (2013) In vivo monitoring of multi-unit neural activity in the suprachiasmatic nucleus reveals robust circadian rhythms in Period $1^{-/-}$mice. PLoS One 8:e64333.

Tischkau SA, Mitchell JW, Tyan SH, Buchanan GF, Gillette MU (2003) Ca2+/cAMP response element-binding protein (CREB)-dependent activation of Perl is required for light-induced signaling in the suprachiasmatic nucleus circadian clock. J Biol Chem 278:718-723.

vanderLeest HT, Vansteensel MJ, Duindam H, Michel S, Meijer JH (2009) Phase of the electrical activity rhythm in the SCN in vitro not influenced by preparation time. Chronobiol Int 26:1075-1089.

Wagner S, Castel M, Gainer H, Yarom Y (1997) GABA in the mammalian suprachiasmatic nucleus and its role in diurnal rhythmicity. Nature 389:598-603.

Xu Y, Toh KL, Jones CR, Shin JY, Fu YH, Ptacek LJ (2007) Modeling of a human circadian mutation yields insights into clock regulation by PER2. Cell 128:59-70.

Yamaguchi S, Isejima H, Matsuo T, Okura R, Yagita K, Kobayashi M, Okamura H (2003) Synchronization of cellular clocks in the suprachiasmatic nucleus. Science 302:1408-1412. 
477

478

479

480

481

482

483

484

485

486

487

488

489

490

491

492
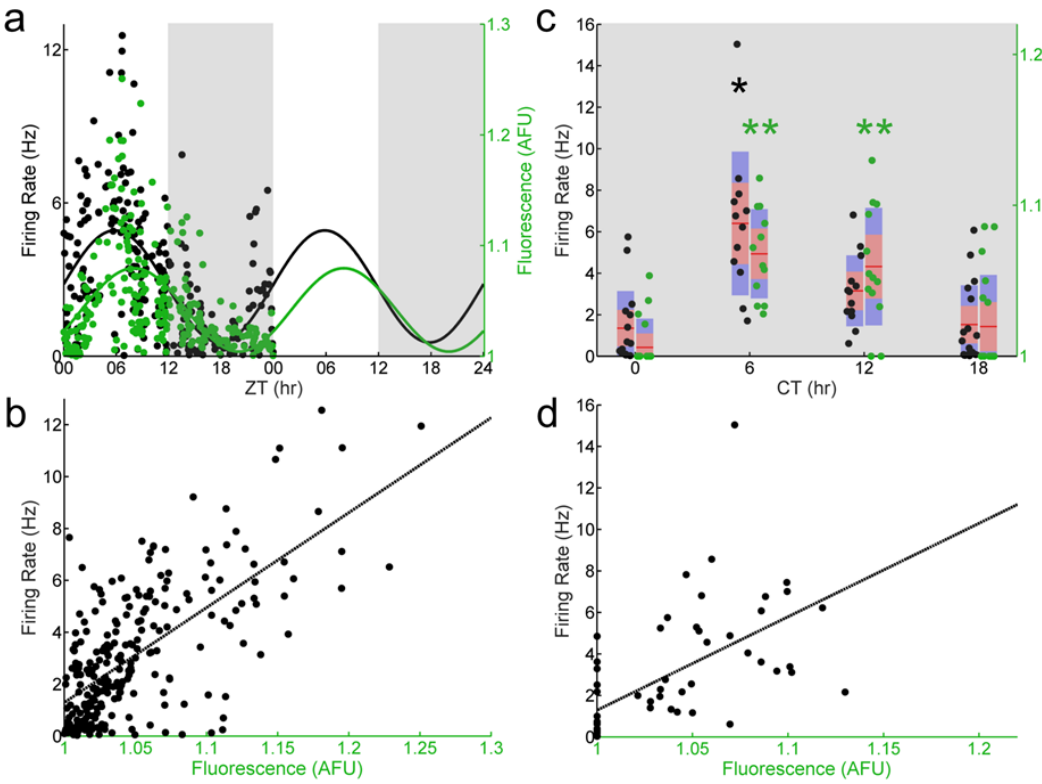

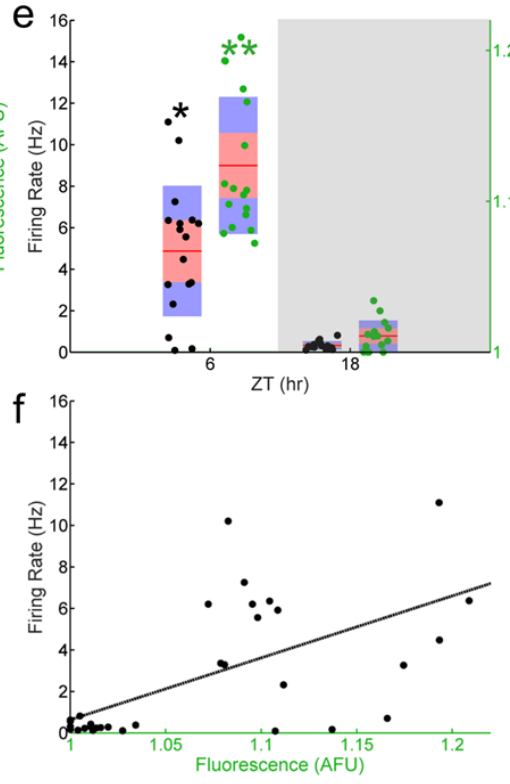

Figure 1: E-box driven gene expression and spontaneous firing rate are rhythmic and correlated in individual SCN neurons.

(a) LD (light, white shading; dark, gray shading) firing rates (black dots) and fluorescence intensities (green dots) from individual Perl::GFP SCN neurons recorded throughout the day (n $=249$ cells, 34 mice). Population rhythmicity determined using a Cosinor fit; firing rate (black line), $\mathrm{r}^{2}=0.3267, \mathrm{p}<0.001$; fluorescence intensity (green line), $\mathrm{r}^{2}=0.2985, \mathrm{p}<0.001$. The Cosinor fit has been extrapolated past 24 hours to better allow visualization of the phase relationship between the firing rate and fluorescence intensity curves. (b) Firing rate versus fluorescence intensity in individual Perl::GFP SCN neurons recorded throughout the day in LD show a positive correlation $\left(n=249\right.$ cells, 34 mice; Pearson's $\left.r, r^{2}=0.4504, p<0.001\right)$. (c) DD (gray shading) firing rates (black dots) and fluorescence intensities (green dots) from individual Per $1::$ GFP SCN neurons recorded throughout the day $(\mathrm{n}=58$ cells, 10 mice). Population rhythmicity determined using a Kruskal-Wallis ANOVA on Ranks test (*, firing rate, $\mathrm{p}<0.001$; **, fluorescence, $\mathrm{p}<0.001)$. Points are overlaid with red $(95 \%$ confidence interval) and blue bars (one standard deviation). (d) Firing rate versus fluorescence intensity in individual 
493 Per $1::$ GFP SCN neurons recorded throughout the day in DD show a positive correlation $(\mathrm{n}=58$

494 cells, 10 mice; Pearson's r, $\mathrm{r}^{2}=0.3621, \mathrm{p}<0.001$ ). (e) LD firing rates (black dots) and

495 fluorescence intensities (green dots) from individual Perl::GFP SCN neurons recorded

496 throughout the day in the presence of GABAzine $(n=32$ cells, 4 mice). Population rhythmicity

497 determined using a Mann-Whitney $U$ test $\left(^{*}\right.$, firing rate, $\mathrm{p}<0.001$; **, fluorescence, $\left.\mathrm{p}<0.001\right)$.

498 Points are overlaid with red (95\% confidence interval) and blue bars (one standard deviation). (f)

499 Firing rate versus fluorescence intensity in individual Per $1:$ GFP SCN neurons recorded

500 throughout the day in LD in the presence of GABAzine show a positive correlation $(\mathrm{n}=32$ cells,

5014 mice; Pearson's r, $\left.\mathrm{r}^{2}=0.5744, \mathrm{p}<0.001\right)$. 
502
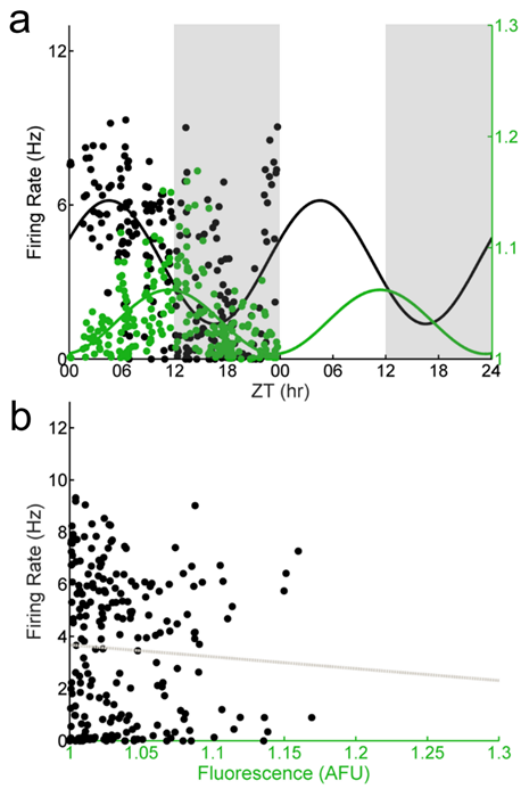
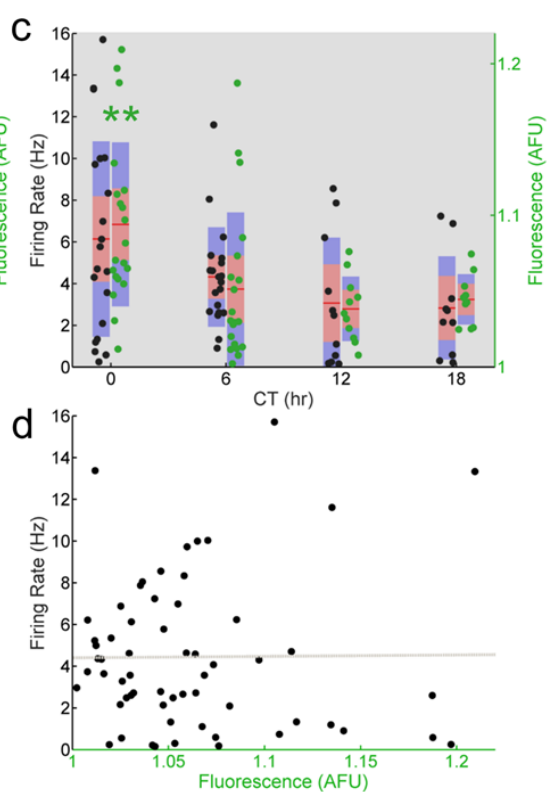

503

504

505

506

507

508

509

510

511

512

513

514

515

516

Figure 2: E-box driven gene expression and spontaneous firing rate are not correlated in

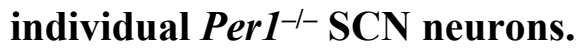

(a) LD (light, white shading; dark, gray shading) firing rates (black dots) and fluorescence intensities (green dots) from individual $\mathrm{Per}^{-1-}$; Perl::GFP SCN neurons recorded throughout the day $(n=227$ cells, 21 mice). Population rhythmicity determined using a Cosinor fit; firing rate (black line), $\mathrm{r}^{2}=0.3988, \mathrm{p}<0.001$; fluorescence intensity (green line), $\mathrm{r}^{2}=0.2822, \mathrm{p}<0.001$. The Cosinor fit has been extrapolated past 24 hours to better allow visualization of the phase relationship between the firing rate and fluorescence intensity curves. (b) Firing rate versus fluorescence intensity in individual $\mathrm{Perl}^{-/-} \mathrm{SCN}$ neurons recorded throughout the day in LD are not correlated $\left(n=227\right.$ cells, 21 mice; Pearson's $\left.r, r^{2}=0.0032, p=0.3968\right)$. (c) DD (gray shading) firing rates (black dots) and fluorescence intensities (green dots) from individual Per $1^{-/-}$ $\mathrm{SCN}$ neurons recorded throughout the day $(\mathrm{n}=61$ cells, 7 mice $)$. Population rhythmicity determined using a Kruskal-Wallis ANOVA on Ranks test $(* *$, fluorescence, $\mathrm{p}<0.001)$. Points are overlaid with red (95\% confidence interval) and blue bars (one standard deviation). (d) Firing 
517 rate versus fluorescence intensity in individual $\mathrm{Perl}^{-/-} \mathrm{SCN}$ neurons recorded throughout the day

518 in DD are not correlated $\left(n=61\right.$ cells, 7 mice; Pearson's $\left.r, r^{2}=0.0049, p=0.4831\right)$. 
519
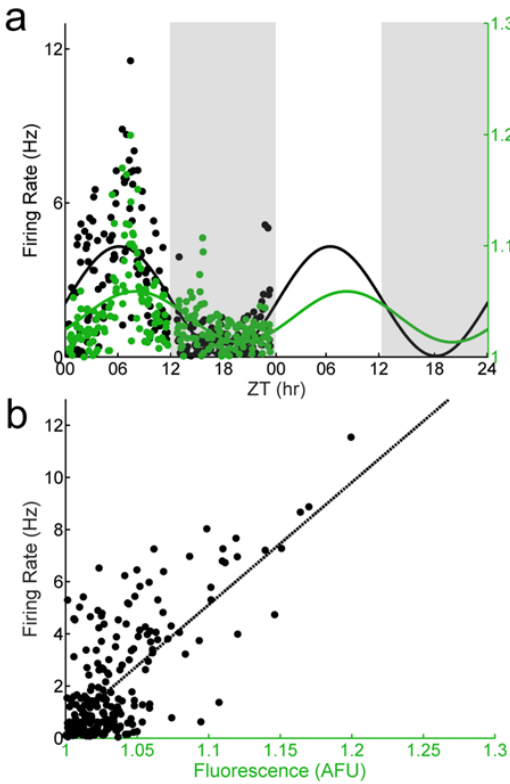
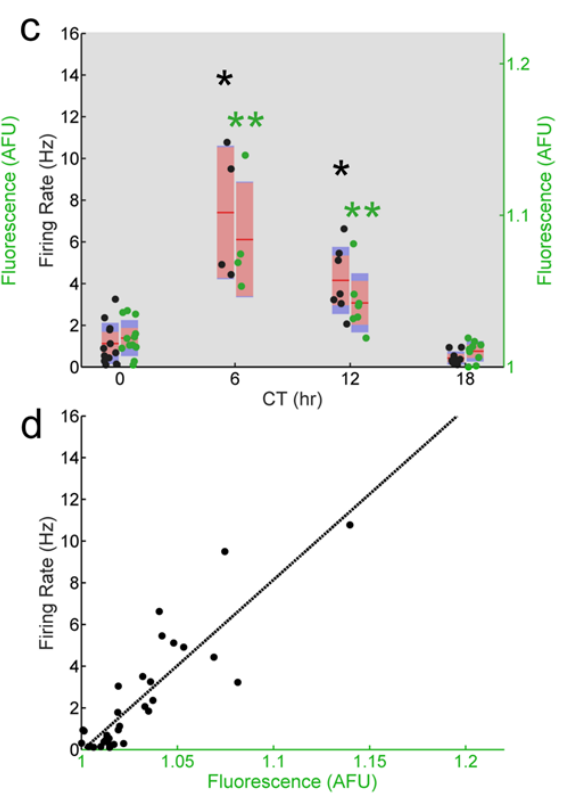

Figure 3: The correlation between E-box driven gene expression and spontaneous firing rate is preserved in individual $\operatorname{Per}^{-1-}$ SCN neurons.

522

(a) LD (light, white shading; dark, gray shading) firing rates (black dots) and fluorescence intensities (green dots) from individual $P e r 2^{--}$; Per $1::$ GFP SCN neurons recorded throughout the day $(n=230$ cells, 10 mice). Population rhythmicity determined using a Cosinor fit; firing rate (black line), $\mathrm{r}^{2}=0.4951, \mathrm{p}<0.001$; fluorescence intensity (green line), $\mathrm{r}^{2}=0.2561, \mathrm{p}<0.001$. The Cosinor fit has been extrapolated past 24 hours to better allow visualization of the phase relationship between the firing rate and fluorescence intensity curves. (b) Firing rate versus fluorescence intensity in individual $\mathrm{Per}^{-{ }^{--}} \mathrm{SCN}$ neurons recorded throughout the day in LD show a positive correlation $\left(n=230\right.$ cells, 10 mice; Pearson's $\left.r, r^{2}=0.6328, p<0.001\right)$. (c) DD (gray shading) firing rates (black dots) and fluorescence intensities (green dots) from individual Per $2^{--}$SCN neurons recorded throughout the day $(\mathrm{n}=32$ cells, 7 mice). Population rhythmicity determined using a Kruskal-Wallis ANOVA on Ranks test ${ }^{*}$, firing rate, $\mathrm{p}<0.001$; **, fluorescence, $\mathrm{p}<0.001)$. Points are overlaid with red $(95 \%$ confidence interval) and blue bars 534 (one standard deviation). (d) Firing rate versus fluorescence intensity in individual Per2 $^{-/-}$SCN 
535 neurons recorded throughout the day in DD show a positive correlation $(n=32$ cells, 7 mice;

536 Pearson's $\left.\mathrm{r}, \mathrm{r}^{2}=0.7572, \mathrm{p}<0.001\right)$. 


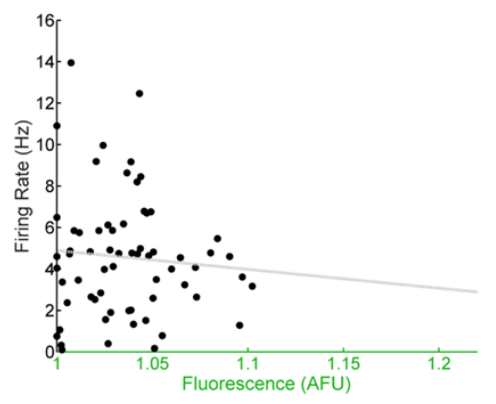

538 Figure 4: E-box driven gene expression and spontaneous firing rate are not correlated in individual Per1 $^{-/-} ;$Per2 $^{-/-}$SCN neurons.

540 Firing rate versus fluorescence intensity in individual $\operatorname{Per}^{-1-} ; \mathrm{Per}^{-/-} \mathrm{SCN}$ neurons recorded

541 throughout the day in DD are not correlated $\left(n=65\right.$ cells, 6 mice; Pearson's $r, r^{2}=0.0036, p=$ $5420.5146)$. 

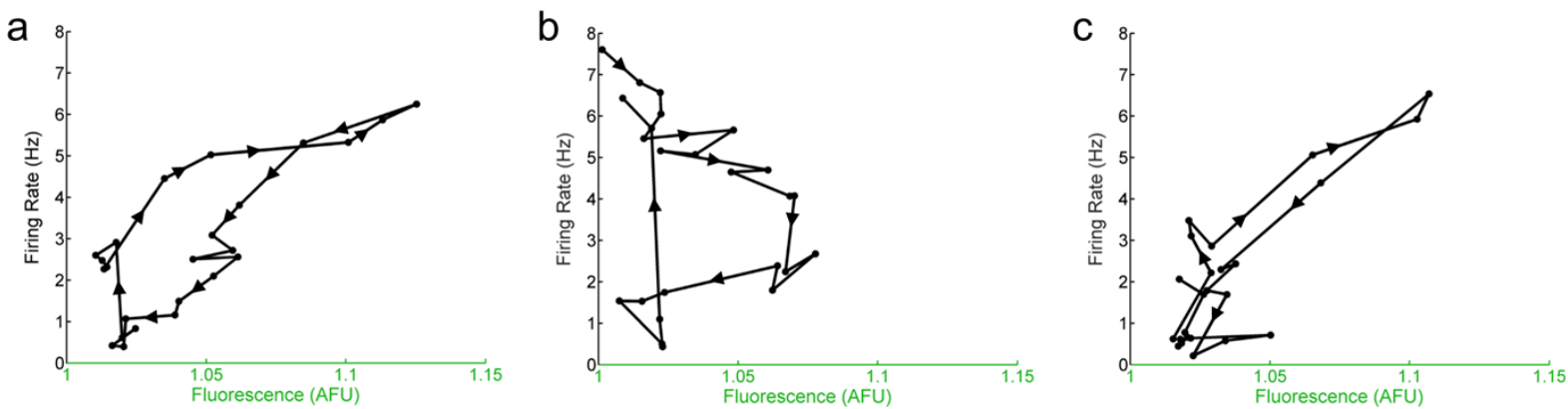

Figure 5: The hourly relationship between E-box driven gene expression and spontaneous firing rate is cyclical.

547

(a) Mean hourly firing rate and fluorescence intensities (black dots) of Per 1::GFP SCN neurons recorded throughout the day in LD. (b) Mean hourly firing rate and fluorescence intensities (black dots) of $\mathrm{Perl}^{-/-} \mathrm{SCN}$ neurons recorded throughout the day in LD. (c) Mean hourly firing rate and fluorescence intensities (black dots) of $\mathrm{Per}^{-/-} \mathrm{SCN}$ neurons recorded throughout the day in LD. In (a-c), arrows and lines represent the direction of the progression of the time of day of recording (i.e., from ZT 0 to ZT 23). 